\title{
ARTICLE
}

Received 30 Jan 2014 | Accepted 23 May 2014 | Published 24 Jun 2014 DOl: 10.1038/ncomms5208

\section{Electrical control of large magnetization reversal in a helimagnet}

Yi Sheng Chai ${ }^{1, \star}$, Sangil Kwon ${ }^{2, \star}$, Sae Hwan Chun ${ }^{1}$, Ingyu Kim, Byung-Gu Jeon ${ }^{1}$, Kee Hoon Kim ${ }^{1,3}$ \& Soonchil Lee ${ }^{2}$

Reversal of magnetization $M$ by an electrical field $E$ has been a long-sought phenomenon in materials science because of its potential for applications such as memory devices. However, the phenomenon has rarely been achieved and remains a considerable challenge. Here we report the large $M$ reversal by $E$ in a multiferroic $\mathrm{Ba}_{0.5} \mathrm{Sr}_{1.5} \mathrm{Zn}_{2}\left(\mathrm{Fe}_{0.92} \mathrm{Al}_{0.08}\right)_{12} \mathrm{O}_{22}$ crystal without any external magnetic field. Upon sweeping $E$ through the range of $\pm 2 \mathrm{MVm}^{-1}, M$ varied quasi-linearly in the range of $\pm 2 \mu_{B}$ per f.u., resulting in the $M$ reversal. Strong electrical modulation of $M$ at zero magnetic field were observable up to $\sim 150 \mathrm{~K}$. Nuclear magnetic resonance measurements provided microscopic evidence that the electric field and the magnetic field play equivalent roles in modulating the volume of magnetic domains. Our results suggest that the soft ferrimagnetism and the associated transverse conical state are key ingredients to achieve the large magnetization reversal at fairly high temperatures.

\footnotetext{
${ }^{1}$ Center for Novel States of Complex Material Research (CeNSCMR), Department of Physics and Astronomy, Seoul National University, Seoul 151-747, South Korea. ${ }^{2}$ Department of Physics, KAIST, Daejeon 305-701, South Korea. ${ }^{3}$ Institute of Applied Physics, Seoul National University, Seoul 151-747, South Korea. ${ }^{*}$ These authors contributed equally to this work. Correspondence and requests for materials should be addressed to K.H.K. (email: khkim@phya.snu.ac.kr) or to S.L. (email: soonchillee@kaist.ac.kr).
} 
E: lectric-field $(E)$ control of magnetization $(M)$ has certain advantages over current-driven control because of its smaller power consumption. For several decades, the capability of achieving this type of control has been tested in a special class of magnetic insulators, so-called magnetoelectrics (MEs) or multiferroics, in which $M$ (or the electric polarization $P$ ) is expected to be modulated by $E$ (or the magnetic field $H)^{1-3}$. Such cross-coupling effects between the magnetic and electric order parameters can be effectively described in terms of direct and converse magnetoelectric effects ${ }^{4}$ :

$$
P=\alpha H+\frac{\beta H^{2}}{2}+\cdots \text { and } \mu_{0} M=\alpha_{c} E+\frac{\gamma E^{2}}{2}+\cdots
$$

where $\alpha\left(\alpha_{c}\right)$ and $\beta(\gamma)$ represent the linear and quadratic ME (converse ME) coefficients. If any of the above direct ME coefficients is large, significant converse ME effects should be observable, as the two types of effects are roughly proportional to each other. However, most ME and multiferroic compounds have thus far exhibited quite small ME coefficients such that the modulation of $M$ by $E$ is too small to be practically useful ${ }^{5-7}$. It is only recently that several multiferroic compounds have demonstrated appreciable modulation of $M$ with enhanced ME coupling coefficients ${ }^{8-10}$.

On the other hand, to better exploit ME multiferroics in practical devices, for example, magnetic information storage, $M$ reversal by $E$ without the application of an $H$-bias becomes a key requirement. Very recently, there has been progress towards achieving this goal. The reversal of ferromagnetic domains has been successfully achieved in a ferromagnet/multiferroic heterostructure at room temperature ${ }^{11}$. However, the observation was limited to interface magnetism. Tokunaga et al. ${ }^{12}$ have demonstrated bulk magnetization reversal in $\mathrm{Dy}_{0.75} \mathrm{Gd}_{0.25} \mathrm{FeO}_{3}$ and $\mathrm{Dy}_{0.7} \mathrm{~Tb}_{0.3} \mathrm{FeO}_{3}$ at $2.5 \mathrm{~K}$, with a maximum modulation of $\sim \pm 0.2 \mu_{\text {B }}$ per f.u. However, full reversal could be achieved only at very low temperatures-below $3 \mathrm{~K}$-because the phenomenon stems from the coupling between rare-earth and Fe moments. Meanwhile, magnetization reversal has been demonstrated in an archetypal $\mathrm{ME} \mathrm{Cr}_{2} \mathrm{O}_{3}$ crystal at room temperature, but the modulation strength was small $\left(10^{-3} \mathrm{emu} \mathrm{g}^{-1}\right.$, equivalent to $\sim 3 \times 10^{-5} \mu_{\text {B }}$ per f.u. $)^{13}$ because of the small ME coefficient, as is typically observed in linear $\mathrm{MEs}^{5}$. Therefore, it is highly desirable to search for a novel mechanism that can drive bulk magnetization reversal with large modulation amplitudes, high switching speeds and high operation temperatures.

To this end, we note that ferrites with hexagonal structures have several advantages. Such systems have long been used in applications such as refrigerator magnets and floppy disks because of their large ferrimagnetic moments and high ordering temperatures ${ }^{14}$. Because of their structural flexibility of the $c$-axis stacking sequence and chemical substitution, numerous hexaferrites with wide structural variants $(\mathrm{M}, \mathrm{Y}, \mathrm{W}, \mathrm{Z}, \mathrm{X}$ and U-types) have been identified, and more may potentially be found. More interestingly, when structure and spin frustration are properly controlled, it has been rather recently found that many of these hexaferrites can become ME with stabilization of a helical or conical spin ordering ${ }^{15}$. The first discovery of such ME effects was made by Kimura et al. ${ }^{16}$ in a $\mathrm{Zn}_{2} \mathrm{Y}$-type hexaferrite $\mathrm{Ba}_{0.5} \mathrm{Sr}_{1.5} \mathrm{Zn}_{2} \mathrm{Fe}_{12} \mathrm{O}_{22}$, in which field-induced $P$ was observed at the magnetic field around $1 \mathrm{~T}$. It was subsequently found that the critical magnetic field necessary to induce $P$ became substantially lowered $(\sim 1-10 \mathrm{mT})$ in $\mathrm{Mg}_{2} \mathrm{Y}$-type ${ }^{17}$ or Al-doped $\mathrm{Zn}_{2} \mathrm{Y}$-type hexaferrites, resulting in giant ME coupling ${ }^{18}$. Furthermore, direct ME effects were also observed at fairly high temperatures in M-type hexaferrites ${ }^{19}$ and even at room temperature in $\mathrm{Co}_{2} \mathrm{Z}$ type hexaferrites ${ }^{20}$. Later on, direct ME effects were also found in the new $\mathrm{U}$ - and $\mathrm{W}$-type hexaferrites ${ }^{21,22}$. Therefore, upon further control of the material properties of these electrically active hexaferrites, it is expected that large converse ME effects may potentially be achieved.

In the case of the converse ME effect, strong $M$ modulation by $E$ and four non-volatile $M$ states were first observed at room temperature in a single crystal of $\mathrm{Co}_{2} \mathrm{Z}$-type hexaferrite, in which the maximum direct ME coupling constants were $\sim 3,200$ and $\sim 150 \mathrm{ps} \mathrm{m}^{-1}$ at finite $(=10.5 \mathrm{mT})$ and zero $H$-bias, respectively ${ }^{10}$. The modulation of $M$ by $E$ was indeed observed without $H$-bias at room temperature, but the ME effect was dominantly quadratic, so the reversal of $M$ could not be achieved. Very recently, converse ME effects have also been reported at room temperature in polycrystalline $\mathrm{Co}_{2} \mathrm{Y}$-type hexaferrites with a maximum converse ME coupling constant of $\sim 400 \mathrm{ps} \mathrm{m}^{-1}$ at an $H$-bias of $1.3 \mathrm{mT}^{23}$. Although nearly linear modulation of $M$ by $E$ was observed, the reversal of $M$ by $E$ could not be achieved without invoking an $H$-bias. Therefore, to date, the true reversal of $M$ by $E$ without any bias magnetic field has not been achieved in hexaferrites, presumably because of the dominant quadratic modulation and the small ME coupling constants. Moreover, the microscopic mechanism for the realization of large converse ME effects in hexaferrites remains unknown.

Here we report the realization of strong modulation and large reversal of magnetization by $E$ in a multiferroic $\mathrm{Ba}_{0.5} \mathrm{Sr}_{1.5} \mathrm{Zn}_{2}\left(\mathrm{Fe}_{0.92} \mathrm{Al}_{0.08}\right)_{12} \mathrm{O}_{22}$ crystal at zero $H$, in which a transverse conical spin state exhibits remanent $M$ and $P$ below $\sim 150 \mathrm{~K}$. The strong modulation of $M$ in the range of $\pm 2 \mu_{\mathrm{B}}$ per f.u. under the electric-field variation of $\pm 2 \mathrm{MV} \mathrm{m}^{-1}$ was achieved, clearly resulting in the reversal of $M$ up to $\sim 100 \mathrm{~K}$. Moreover, the remanent $M$ exhibited non-volatile changes of $\pm 0.11-0.15 \mu_{\mathrm{B}}$ per f.u., depending on the history of the applied electric field up to $\sim 150 \mathrm{~K}$. NMR measurements showed microscopic evidence that the electric field and the magnetic field play equivalent roles in modulating the volume of magnetic domains.

\section{Results}

Basic properties of $\mathbf{B a}_{0.5} \mathrm{Sr}_{1.5} \mathbf{Z n}_{\mathbf{2}}\left(\mathrm{Fe}_{\mathbf{0 . 9 2}} \mathrm{Al}_{\mathbf{0 . 0 8}}\right)_{12} \mathrm{O}_{22} \cdot \mathrm{Ba}_{0.5} \mathrm{Sr}_{1.5}$ $\mathrm{Zn}_{2}\left(\mathrm{Fe}_{0.92} \mathrm{Al}_{0.08}\right)_{12} \mathrm{O}_{22}$ consists of a series of tetrahedral and octahedral $\mathrm{Fe} / \mathrm{Zn}$ layers stacked along the $c$-axis $(\|[001])^{24}$, as shown in Fig. 1a. Under zero-field cooling below $60 \mathrm{~K}$, it is known
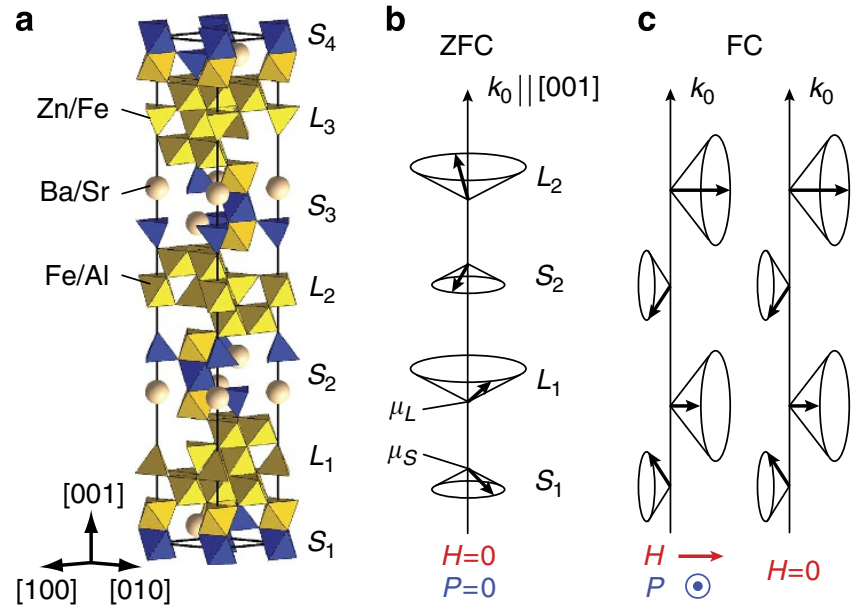

Figure 1 | Crystal and magnetic structures of $\mathbf{B a}_{0.5} \mathbf{S r}_{1.5} \mathbf{Z n}_{\mathbf{2}}$ $\left(\mathbf{F e} \mathbf{0 . 9 2}_{\mathbf{0 . 9}} \mathbf{A l}_{\mathbf{0 . 0 8}}\right)_{12} \mathbf{O}_{\mathbf{2 2}}$. Schematic diagrams of (a) the crystal structure, (b) the longitudinal conical spin state formed after zero-field cooling (ZFC), and (c) the transverse conical spin state formed after field cooling (FC). $\boldsymbol{\mu}_{L}$ and $\boldsymbol{\mu}_{S}$ denote the net magnetic moments in the $L$ and $S$ blocks, respectively, and $\mathbf{k}_{0}$ is the spin modulation vector. 
a

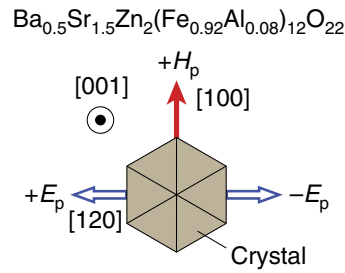

C
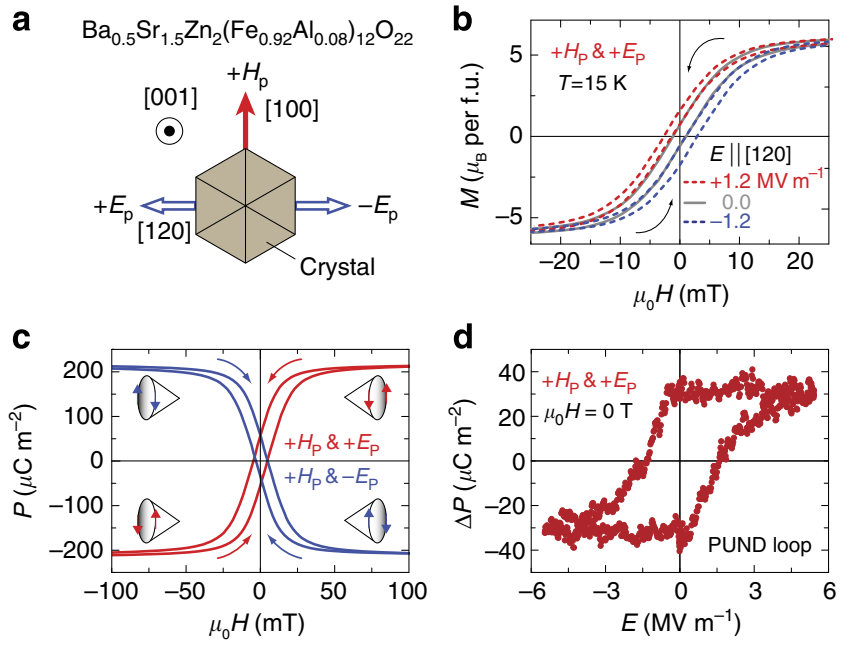

Figure 2 | Basic properties of $\mathrm{Ba}_{0.5} \mathrm{Sr}_{1.5} \mathrm{Zn}_{2}\left(\mathrm{Fe}_{0.92} \mathrm{Al}_{0.08}\right)_{12} \mathrm{O}_{22}$ after the magnetoelectric annealing procedures. (a) $\mathrm{A}$ schematic diagram of the $\mathrm{ME}$ annealing schemes of $+H_{\mathrm{p}} \&+E_{\mathrm{p}}$ and $+H_{\mathrm{p}} \&-E_{\mathrm{p}} . H_{\mathrm{p}}$ and $E_{\mathrm{p}}$ denote the magnetic and the electric poling fields applied during the $M E$ annealing procedures, respectively. (b) $M-H$, (c) $P-H$ and (d) $\Delta P-E$ loops of

$\mathrm{Ba}_{0.5} \mathrm{Sr}_{1.5} \mathrm{Zn}_{2}\left(\mathrm{Fe}_{0.92} \mathrm{Al}_{0.08}\right)_{12} \mathrm{O}_{22}$ at $15 \mathrm{~K}$. The spin helicity of the transverse conical state and the resultant electric-polarization direction are most likely determined by the $M E$ annealing conditions, represented by $+H_{\mathrm{p}} \&+E_{\mathrm{p}}$ or $+H_{\mathrm{p}} \&-E_{\mathrm{p}}$ (see Methods for a description of the procedures). The $\triangle P-E$ hysteresis loop, based on the Positive-Up-Negative-Down (PUND) method, indicates that the sample is ferroelectric at $H=0$ after being subjected to ME annealing $\left(+H_{\mathrm{p}} \&+E_{\mathrm{p}}\right)$.

from neutron-diffraction studies ${ }^{25}$ that the compound exhibits an incommensurate longitudinal conical ordering, in which the alternating small $(S)$ and large $(L)$ magnetic blocks have antiparallel $c$-axis components and in-plane helical ordering (Fig. 1b). On the other hand, under field-cooling process or after the application of $\mu_{0} H \geq 0.1 \mathrm{~T}$ subsequent to the zero-field cooling, a transverse conical state with a commensurate modulation vector $\mathbf{k}_{0}=(0,0,3 / 2)$ forms (Fig. $1 \mathrm{c}$, left). It was also found that the transverse conical state formed at finite $H$ still remains as a metastable phase even after the external $H$ is removed (Fig. 1c, right). After such an ME annealing procedure (see Methods and Fig. 2a), the material thus becomes multiferroic, exhibiting remanent $M$ and $P$-values in the $M-H$ (Fig. 2b) and $P-H$ (Fig. 2c) hysteresis loops (P (\|[120]) $\perp \mathbf{M}$ ( $\mid[100]$ ) and also in the $P-E$ hysteresis loop (Fig. 2d). The ferroelectric phase diagram of this material is summarized in Supplementary Fig. 1.

In the $M-H$ hysteresis loops, $M$ is easily saturated and changes sign at small $H$ values of $\sim \pm 30 \mathrm{mT}$ with a small coercive field of $\sim 2 \mathrm{mT}$, indicating that $\mathrm{Ba}_{0.5} \mathrm{Sr}_{1.5} \mathrm{Zn}_{2}\left(\mathrm{Fe}_{0.92} \mathrm{Al}_{0.08}\right)_{12} \mathrm{O}_{22}$ is a very soft ferrimagnet in the transverse conical state. Very small magnetic anisotropy in the $a b$-plane is also confirmed by the angular dependence of $P$ under the horizontal rotation of $H$ (see Methods and Supplementary Fig. 2). The $P-H$ hysteresis loops also demonstrate the saturation and sign change of $P$ at $\sim \pm 40 \mathrm{mT}$, which is similar to the scale of the field required for $M$ reversal. The overall shapes of the $M-H$ and $P-H$ loops thus appear similar to each other. The observations suggest that the soft ferrimagnetic domains and the associated ferroelectric domains must coincide each other, as has similarly been claimed to occur in $\mathrm{CoCr}_{2} \mathrm{O}_{4}$ (ref. 26).

In the $P-H$ hysteresis loops, it is also apparent that a saturated $P$ changes sign upon a change in the initial direction of electric poling $\left(E_{\mathrm{p}}\right)$ during the ME annealing procedure (Fig. 2a). The
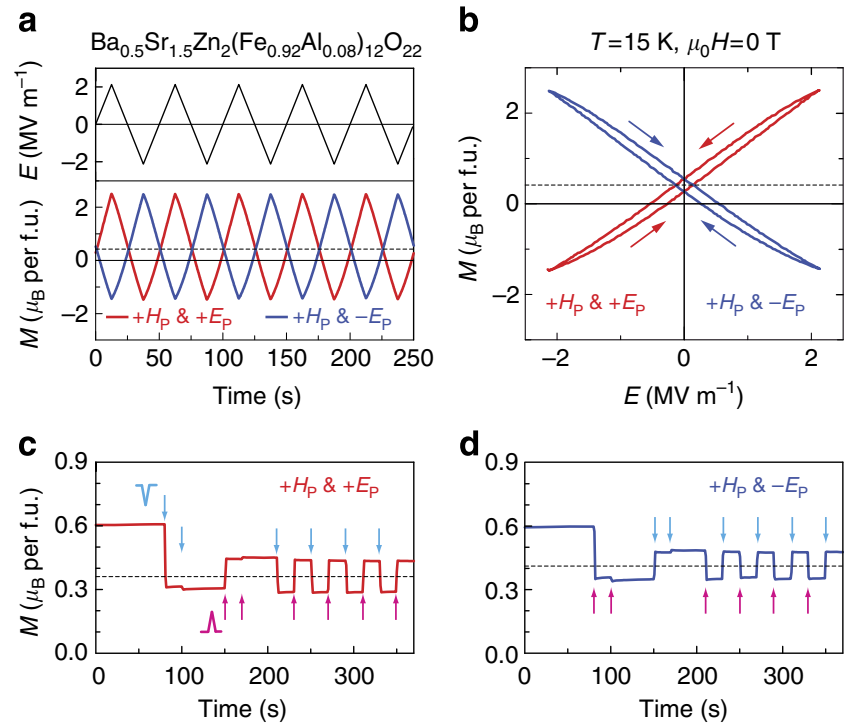

Figure 3 | Repeatable, strong modulation and large reversal of magnetization by electric fields. (a) Periodic modulations of $\mathbf{M} \|[100]$ at zero magnetic field under repeating triangular waves of $\mathbf{E} \|[120]$ after the $M E$ annealing. (b) The resultant $M-E$ loops illustrating the reversal; the magnetization was initially at $\sim 0.6 \mu_{\mathrm{B}}$ per f.u. because of the $+H_{\mathrm{p}}$ condition, and its average value was $\sim 0.4 \mu_{\mathrm{B}}$ per f.u. (dotted lines). (c,d) Jumps in the remanent $M$ caused by a short, triangular electric pulse with either a positive (red arrows) or negative (blue arrows) peak value of 6.8 $\mathrm{MVm}^{-1}$ and a duration of $0.5 \mathrm{~ms}$ for the two $\mathrm{ME}$ annealing conditions of (c) $+H_{\mathrm{p}} \&+E_{\mathrm{p}}$ and $(\mathbf{d})+H_{\mathrm{p}} \&-E_{\mathrm{p}}$. The magnetization, which was measured for $\sim 0.1 \mathrm{~s}$ per point, appears to have changed immediately upon application of the short electric pulse, and the recorded information regarding the pulse history is non-volatile.

origins of such a finite $P$, which changes in sign with varying $H$-bias and $E_{\mathrm{p}}$, and the $\mathbf{P} \perp \mathbf{M}$ relation can be qualitatively understood via the spin current $\operatorname{model}^{27,28}$, in which $\mathbf{P} \propto \mathbf{k}_{0} \times\left(\boldsymbol{\mu}_{S} \times \boldsymbol{\mu}_{L}\right)$, where $\boldsymbol{\mu}_{S}$ and $\boldsymbol{\mu}_{L}$ are the net moments of the adjacent $S$ and $L$ spin blocks, respectively. The sign-changing behaviour of $\mathbf{P}$ with variations in the $E_{\mathrm{p}}$ direction is therefore likely to induce different spin helicities, defined as $\Sigma \boldsymbol{\mu}_{S} \times \boldsymbol{\mu}_{L}$, because the $E_{\mathrm{p}}$ direction can energetically fix the local spin current direction, $\boldsymbol{\mu}_{S} \times \boldsymbol{\mu}_{L}$. It is also important to note that the nearly linear variation of $P$ at a small $H$-bias facilitates the production of the highest ME coefficient $(\mathrm{d} P / \mathrm{d} H)$ of $\sim 15,000 \mathrm{ps} \mathrm{m}^{-1}$ at zero $H$ among bulk multiferroic compounds $^{18}$. Therefore, it is expected that a large converse ME effect should be observed in this compound, although no experimental investigation has yet been conducted.

Electrical modulation and reversal of $M$ at low temperature. Figure $3 \mathrm{a}$ demonstrates that a giant $M$ modulation can be induced by the application of $E$ at zero $H$-bias in a $\mathrm{Ba}_{0.5} \mathrm{Sr}_{1.5} \mathrm{Zn}_{2}$ $\left(\mathrm{Fe}_{0.92} \mathrm{Al}_{0.08}\right)_{12} \mathrm{O}_{22}$ single crystal. Before any experiment was performed, the transverse conical phase was first stabilized at zero $H$-bias after $\mathrm{ME}$ annealing with the $+H_{\mathrm{p}} \&+E_{\mathrm{p}}$ condition. Then, a slowly varying $\mathbf{E} \|[120]$ in the range of $\pm 2 \mathrm{MV} \mathrm{m}^{-1}$ was repeatedly applied with a period of $40 \mathrm{~s}$, while the magnetization $\mathbf{M} \|[100]$ was recorded using a vibrating sample magnetometer to measure the magnetization at time intervals of $0.1 \mathrm{~s}$. We observed a gigantic change in $M$ that was approximately proportional to the change in $E$, with a modulation of $\Delta M \sim \pm 2 \mu_{\mathrm{B}}$ per f.u. for the sweep of $E$ through the range of $\pm 2 \mathrm{MV} \mathrm{m}^{-1}$ (Fig. 3a). It is noteworthy that this modulation of $\Delta M \sim \pm 2 \mu_{\mathrm{B}}$ per f.u. represents the largest modulation magnitude 
ever observed in a bulk compound. The average value of $M$ at zero $H$-bias in this repeated measurement was $\sim 0.4 \mu_{\mathrm{B}}$ per f.u., indicating that $M$ varied between 2.4 and $-1.6 \mu_{\mathrm{B}}$ per f.u., demonstrating the unambiguous reversal of the bulk magnetization direction. The quasi-linear modulation of $M$ was repeatable for many cycles of the $E$ sweep, so the resultant $M-E$ loop was quite reproducible (Fig. 3b). Interestingly, the $M-E$ loops exhibited a small hysteresis, resulting in two stable remanent magnetization $M_{\mathrm{r}}$ values ( 0.47 and $0.33 \mu_{\mathrm{B}}$ per f.u.) at zero $E$-bias. Upon changing the $\mathrm{ME}$ annealing condition to $+H_{\mathrm{p}} \&-E_{\mathrm{p}}$, the $M$ exhibited a quasi-linear decrease with increasing $E$ (blue lines in Fig 3a,b), but the full hysteresis loop retained the same two $M_{\mathrm{r}}$ values. In other words, $M$ clearly exhibited two non-volatile $M_{\mathrm{r}}$ states at zero $E$-bias, the values of which depended on the history of the $E$ sweep and also the sign of $E_{\mathrm{p}}$ in the ME annealing procedure.

Switching of remanent magnetization by electric pulses. Figure $3 \mathrm{c}$ further demonstrates that the two $M_{\mathrm{r}}$ values could be controlled by the application of short $E$ pulses. Figure $3 c$ presents the trace of the $M_{\mathrm{r}}$ values, which was initially $0.6 \mu_{\mathrm{B}}$ per f.u. after $\mathrm{ME}$ annealing under the $+H_{\mathrm{p}} \&+E_{\mathrm{p}}$ condition. Upon the application of a short negative pulse of a triangular $E$ wave with a peak value of $6.8 \mathrm{MV} \mathrm{m}^{-1}$ and a duration of $0.5 \mathrm{~ms}$, the $M_{\mathrm{r}}$ value suddenly dropped to $0.3 \mu_{\mathrm{B}}$ per f.u. The drop in $M_{\mathrm{r}}$ caused by the first $E$ pulse was relatively larger. The second negative pulse induced only a negligible drop in $M_{\mathrm{r}}$, whereas the subsequent positive pulse caused $M_{\mathrm{r}}$ to sharply increase once again to $0.45 \mu_{\mathrm{B}}$ per f.u. Thus, the sequential application of $E$ pulses of opposite direction resulted in sharp jumps of $M_{\mathrm{r}}$ between two independent values centred near $0.37 \mu_{\mathrm{B}}$ per f.u., whereas the latter of two successive $E$ pulses of the same sign did not produce any appreciable change in $M_{\mathrm{r}}$. The switching of $M_{\mathrm{r}}$ between the two values was well-reproducible after many $E$ pulses, with no evidence of any fatigue effect.

Figure $3 \mathrm{~d}$ presents the trace of the $M_{\mathrm{r}}$ values when the ME annealing was performed under the $+H_{\mathrm{p}} \&-E_{\mathrm{p}}$ condition. In this case, the effects of the short positive and negative pulses were reversed, but the overall behaviour was quite similar to that in the case of $+H_{\mathrm{p}} \&+E_{\mathrm{p}}$ condition. The $M_{\mathrm{r}}$ values switched between $0.35 \mu_{\mathrm{B}}$ per f.u. and $0.47 \mu_{\mathrm{B}}$ per f.u., centred on a value of $0.41 \mu_{\mathrm{B}}$ per f.u. The ability to induce such non-volatile $M_{\mathrm{r}}$ changes through the application of short $E$ pulses implies that the ferroelectric and ferrimagnetic domain configurations at zero $H$-bias can be tuned by an external electric field, possibly via the microscopic ME coupling inherent to the investigated hexaferrite. It is also noteworthy that the two non-volatile $M_{\mathrm{r}}$ values and the ability to switch the remanent magnetism between these two values using short electric pulses could be observed even at higher temperatures.

Electrical control of magnetization at higher temperatures. Figure 4 summarizes the $M-E$ hysteresis loops (Fig. 4a) and the variation in $M_{\mathrm{r}}$ values caused by short $E$ pulses (Fig. $4 \mathrm{~b}$ ) at and above $50 \mathrm{~K}$. The $M-E$ hysteresis loop and the change in $M_{\mathrm{r}}$ under short $E$ pulses were clearly observable up to at least $150 \mathrm{~K}$, although the maximum $\Delta M$ and the magnitude of the $M_{\mathrm{r}}$ jumps systematically decreased with increasing temperature. The temperature window of $150-170 \mathrm{~K}$ is consistent with the region in which the phase diagram indicates the stabilization of the transverse conical state only at finite magnetic fields (see Supplementary Fig. 1). In particular, at $T=170 \mathrm{~K}$, the $M-E$ loop evolved into a combination of linear and quadratic ME behaviours, exhibiting an asymmetric parabolic shape (Fig. 4a). Moreover, the hysteretic behaviour became negligible above this
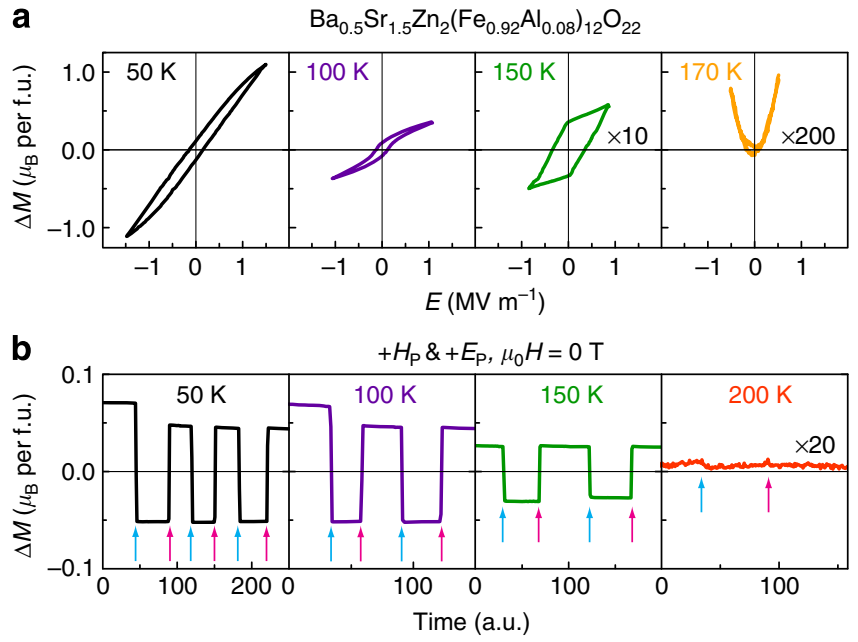

Figure 4 | High-temperature modulation of magnetization under continuous and pulsed electric fields. (a) $\Delta M-E$ loops measured at 50 , 100,150 and $170 \mathrm{~K}$. Hysteretic modulation was clearly observed up to $150 \mathrm{~K}$ (as high as $160 \mathrm{~K}$ ). The central magnetization values at each temperature were found to be $\sim 0.45,0.48,0.10$, and $0.02 \mu_{B}$ per f.u., respectively, and this value was subtracted in each plot to more clearly display the magnetisation behaviour. (b) The non-volatile change in remanent $M$ after the application of a short, triangular electric pulse with either a positive (red arrows) or negative (blue arrows) peak value of $4.2 \mathrm{MVm}^{-1}$, demonstrating that the $M$ jumps occurred up to at least $150 \mathrm{~K}$. The zero value at $200 \mathrm{~K}$ corresponds to the central magnetization value of $0.03 \mu_{\mathrm{B}}$ per f.u.

temperature, and eventually at $200 \mathrm{~K}$, no change in $M_{\mathrm{r}}$ could be observed (Fig. 4b). Therefore, a clear $M-E$ hysteresis loop and non-volatile changes in $M_{\mathrm{r}}$ seem to occur only when the transverse conical states is stabilized at zero $H$-bias. If the temperature to stabilize the transverse conical states at zero $H$-bias can be enhanced further, it is likely that the converse ME effects and its related mechanism, as observed here, could also be achieved even at higher temperatures.

The $M-H$ loops and ${ }^{57} \mathrm{Fe}$ NMR studies under electric-field bias. To demonstrate the changing of $M$ by $E$ under finite $H$, we also measured the $M-H$ loops under an E-bias, as shown in Fig. 2b. Overall, the magnetization curve varied under the application of a non-zero $E$ bias for any given magnetic field, but the relative change was close to maximum at zero magnetic field. The coercive field under finite $E$ was slightly reduced by $\sim 10 \%$, and the $M-H$ curves slightly expanded sideways. Most notably, the $M-H$ curves in Fig. $2 \mathrm{~b}$ were found to be shifted by $\sim \mp 2 \mathrm{mT}$ by an $E$ of $\pm 1.2 \mathrm{MV} \mathrm{m}^{-1}$. These phenomena are very analogous to the usual exchange-bias effect, in which the shift of the central magnetic field occurs as if an internal $H$-bias were applied. In this case, $E$ serves the same role as an internally applied magnetic field in the exchange-bias phenomena, such that a positive (negative) $E$ results in a negative (positive) shift in the hysteresis loop, as if a positive (negative) $H$-bias had already been applied. This result implies that the role of the E-bias is equivalent to that of an $H$-bias itself. Therefore, it is expected that the effects of either an electric field or a magnetic field (although they are perpendicular) in this hexaferrite should be equivalent, and both should affect the clamped ferroelectric/ferrimagnetic domains.

To further understand the microscopic origin of such changes in the $M-H$ loop induced by $E$ and the large converse effects observed in Figs 3 and 4, we systematically studied the 
a

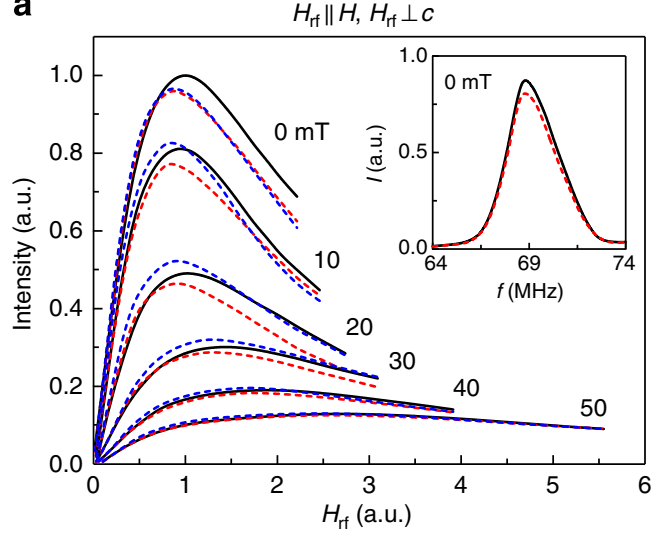

b

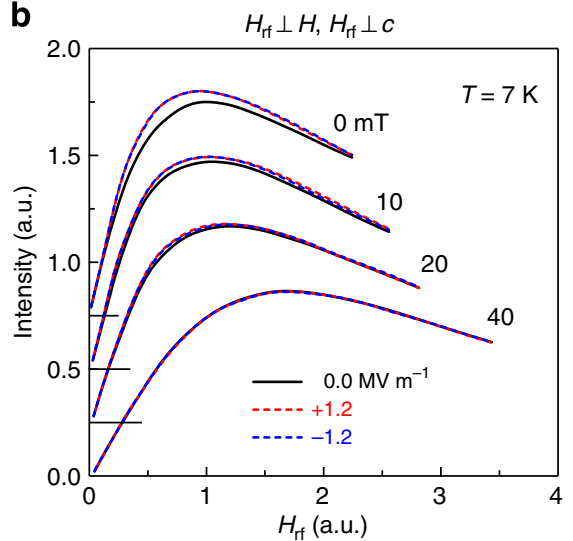

C

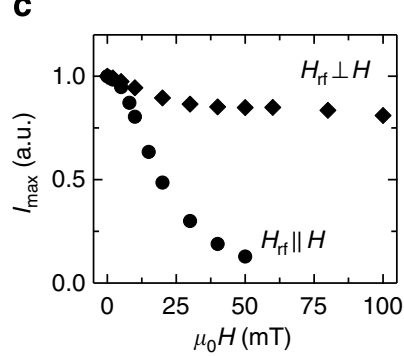

d

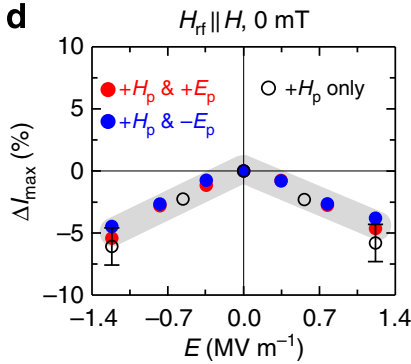

e

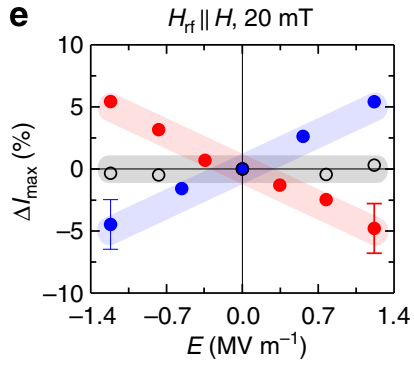

f

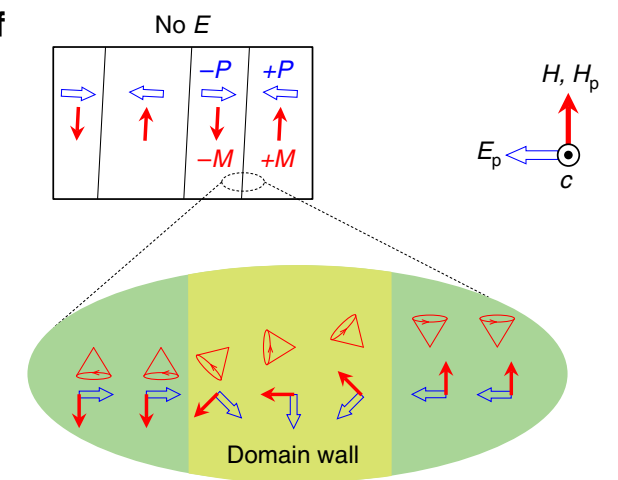

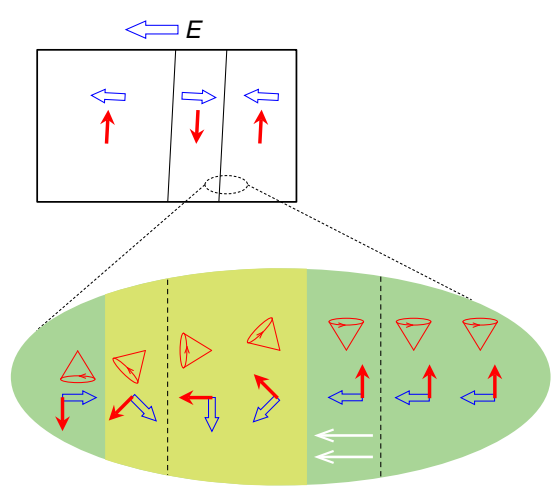

Figure 5 | The effects of magnetic and electric fields on the ${ }^{57} \mathrm{Fe} \mathrm{NMR} \mathrm{signal} \mathrm{and} \mathrm{magnetic} \mathrm{domain} \mathrm{structure} \mathrm{of} \mathrm{Ba}_{0.5} \mathrm{Sr}_{1.5} \mathrm{Zn}_{\mathbf{2}}\left(\mathrm{Fe}_{0.92} \mathrm{Al}_{0.08}\right)_{12} \mathrm{O}_{22}$. The NMR intensity versus $H_{\mathrm{rf}}$ under $+H_{\mathrm{p}} \&+E_{\mathrm{p}}$ condition for (a) $\mathbf{H}_{\mathrm{rf}} \| \mathbf{H}$ and $(\mathbf{b}) \mathbf{H}_{\mathrm{rf}} \perp \mathbf{H}$. The inset of a presents the zero-field ${ }^{57} \mathrm{Fe} N M R$ spectrum. $\ln \mathbf{b}$, the data for $\mu_{0} H=0$ to $20 \mathrm{mT}$ are shifted with respect to one another for clarity. The red dashed curves represent the data acquired under an

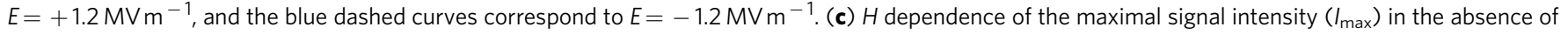
$E$. $(\mathbf{d}, \mathbf{e}) E$ dependence of the relative change in the maximal signal intensity $\left(\Delta I_{\max }\right)$ for $\mathbf{H}_{\mathrm{rf}} \| \mathbf{H}$ at 0 and $20 \mathrm{mT}$. The red and blue solid circles represent data acquired after $+E_{\mathrm{p}}$ and $-E_{\mathrm{p}}$ poling, respectively. The black open circles represent data acquired without $\mathrm{ME}$ annealing. All data were obtained at $7 \mathrm{~K}$. (f) Schematic diagrams of changes in domain configuration with $E$ and $H$ under the $+H_{p} \&-E_{p}$ condition. The directions of the ME annealing fields and the $c$-axis are the same as in Fig. 2a. The two multiferroic domains hold for an orthogonal $\mathrm{M}$ and $\mathrm{P}$ relationship, and their domain walls move under the application of an external $E$ (white solid arrows), thus leading to an increase in the volume of one of multiferroic domains.

enhancement of the ${ }^{57} \mathrm{Fe}$ NMR signal intensity. When nuclear spins are excited by an oscillating magnetic field, they typically precess along the local magnetic field. In magnetic materials, the electron spins coupled with the nuclear spins via the hyperfine interaction in a magnetic domain also precess at the same frequency. This phenomenon generates an oscillating magnetic field that is much stronger than that generated by the nuclear spins only, resulting in an enhanced NMR signal ${ }^{29}$. The signal may also be enhanced in the domain wall, but such an enhancement occurs via a slightly different mechanism. Precessing nuclear spins in a domain wall cause the domain wall to be displaced back and forth at the same frequency. Then, the electron spins in the domain wall begin to oscillate, resulting in signal enhancement. Typically, the enhancement effect in the domain wall is larger than that in the domain.
The inset of Fig. 5a presents the ${ }^{57} \mathrm{Fe}$ NMR spectra of $\mathrm{Ba}_{0.5} \mathrm{Sr}_{1.5} \mathrm{Zn}_{2}\left(\mathrm{Fe}_{0.92} \mathrm{Al}_{0.08}\right)_{12} \mathrm{O}_{22}$ with and without $E$-bias at $\mu_{0} H=0 \mathrm{mT}$. The directions of the fields are the same as in Fig. 2a, with an additional radio frequency (rf) magnetic field $H_{\mathrm{rf}}$ along the $a b$ plane. The NMR intensity was observed to change under external electric and magnetic fields, whereas the shape and position of the spectrum were not altered. In Fig. 5a,b, the signal intensities versus $H_{\mathrm{rf}}$ are plotted for various $H$ with and without $E$-bias for $\mathbf{H}_{\mathrm{rf}} \| \mathbf{H}$ and $\mathbf{H}_{\mathrm{rf}} \perp \mathbf{H}$, respectively. The overall intensity of the spectrum decreased with increasing $H$ in both cases, which is consistent with general expectations because the presence of an external magnetic field prevents the magnetic moments of electrons from vibrating freely. However, we found that the enhancement effect was sensitive to the direction of $\mathbf{H}_{\mathrm{rf}}$, as illustrated in Fig. 5c. The signal intensity decreased rapidly with $H$ 

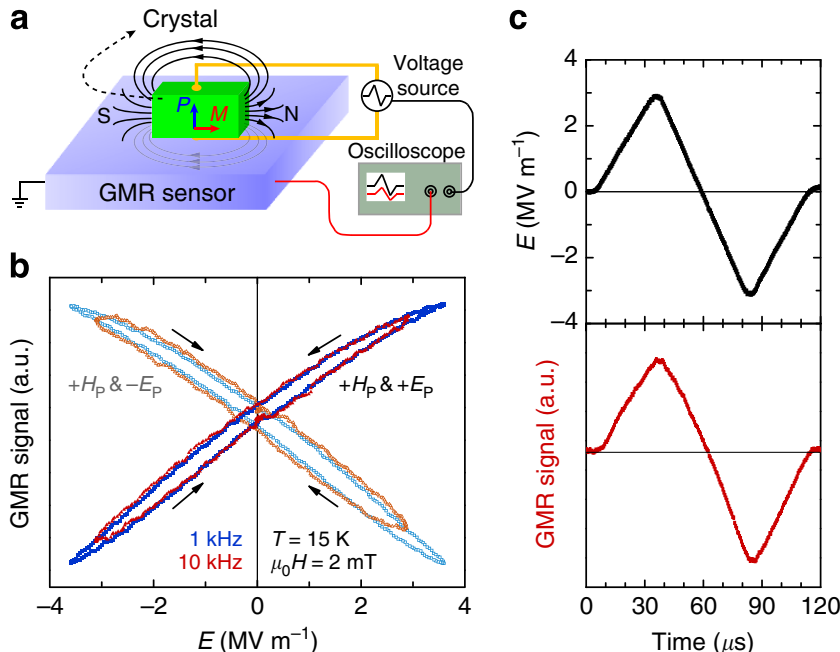

Figure 6 | Measurement of rapid magnetization change using a GMR

sensor. (a) A schematic diagram of a $\mathrm{Ba}_{0.5} \mathrm{Sr}_{1.5} \mathrm{Zn}_{2}\left(\mathrm{Fe}_{0.92} \mathrm{Al}_{0.08}\right)_{12} \mathrm{O}_{22}$ single crystal bonded to the top of a GMR sensor. Electric pulses were applied sequentially while an oscilloscope was used to monitor the time dependence of the electric pulses and the changes in the voltage measured by the GMR sensor. (b) E dependence of the GMR signals at $15 \mathrm{~K}$. The maximum modulation of the GMR signals under the electric-field sweep corresponds to an equivalent change in the magnetic field of $\sim 2.5 \mathrm{mT}$. Note that the hysteresis in the plot of the GMR signal versus the electric field primarily originates from changes in the remanent $M$. (c) The full increase or decrease of the GMR signal occurs within $\sim 30 \mu \mathrm{s}$, limited by the rise or fall time of the electric pulses, thereby indicating that the magnetization jumps can occur at least within $30 \mu$ s and possibly much more rapidly.

and disappeared above $50 \mathrm{mT}$ for $\mathbf{H}_{\mathrm{rf}} \| \mathbf{H}$, whereas it was much less sensitive for $\mathbf{H}_{\mathrm{rf}} \perp \mathbf{H}$. Because $\mathrm{Ba}_{0.5} \mathrm{Sr}_{1.5} \mathrm{Zn}_{2}\left(\mathrm{Fe}_{0.92} \mathrm{Al}_{0.08}\right)_{12} \mathrm{O}_{22}$ is nearly in a single magnetic domain state at $\mu_{0} H=50 \mathrm{mT}$, as can be inferred from Fig. $2 b$, this result suggests that the NMR signal obtained for $\mathbf{H}_{\mathrm{rf}} \| \mathbf{H}$ originated primarily from domain walls and that the signal obtained for $\mathbf{H}_{\mathrm{rf}} \perp \mathbf{H}$ originated primarily from domains.

The enhancement effect due to the rotation of the domain magnetization becomes maximal when $\mathbf{H}_{\mathrm{rf}}$ and the direction of magnetization in the domains are perpendicular to each other. On the other hand, the enhancement due to domain-wall movement is maximal when $\mathbf{H}_{\mathrm{rf}}$ and the direction of magnetization in the domains are parallel to each other because in this case, the domain walls can move more easily ${ }^{30}$. Therefore, the fact that the NMR signal primarily originates from domains for $\mathbf{H}_{\mathrm{rf}} \perp \mathbf{H}$ and from domain walls for $\mathbf{H}_{\mathrm{rf}} \| \mathbf{H}$ means that the direction of magnetization in the domains is mostly parallel or antiparallel to $\mathbf{H}$, and that the domain walls are parallel to $\mathbf{H}$, as depicted in Fig. 5f. It is not surprising because of very small magnetic anisotropy in the plane for the transverse conical state (see Supplementary Fig. 2), although the hexagonal crystal symmetry predicts, in principle, three easy axes in the $a b$ plane $^{31}$.

The most striking finding presented in Fig. 5a,b is that the signal intensity changed with $E$, either increasing or decreasing depending on the presence of $H$ and the polarity of $E$. Fig. 5 d,e illustrates the $E$ dependence of the relative change in the maximal signal intensity $\left(\Delta I_{\max }\right)$. At $\mu_{0} H=0 \mathrm{mT}$ (Fig. $5 \mathrm{~d}$ ), $\Delta I_{\max }$ decreased linearly with increasing $E$, irrespective of the direction of $E_{\mathrm{p}}$. Because the signal intensity is proportional to the domain-wall volume for $\mathbf{H}_{\mathrm{rf}} \| \mathbf{H}$, a decrease in $\Delta I_{\max }$ indicates a domain-wall reduction induced by $E$ (Fig. 5f). The non-poled sample (black open circles) exhibited nearly identical behaviour at $\mu_{0} H=0 \mathrm{mT}$. At $\mu_{0} H=20 \mathrm{mT}$ (Fig. 5e), the application of an $E$ in the opposite direction to $E_{\mathrm{p}}$ partially recovered the intensity reduction caused by $H$, whereas the application of an $E$ in the same direction further reduced the intensity. This behaviour can also be understood as a change in the domain-wall volume: An $E$ in the same direction as the electric poling causes the domains whose orientations are parallel to $H$ to expand, and consequently, the domain-wall volume is reduced. An $E$ in the opposite to the direction of the electric poling produces the opposite effects. For the non-poled sample, $\Delta I_{\max }$ remained nearly constant, regardless of the electric-field direction. This finding suggests that half of the ferrimagnetic domains were coupled to the ferroelectric domains in the positive direction and the other half were coupled to the ferroelectric domains in the negative direction. This result is consistent with the observation that there are two different chiralities in the conical spin ordering of $\mathrm{Ba}_{0.5} \mathrm{Sr}_{1.5} \mathrm{Zn}_{2}$ $\left(\mathrm{Fe}_{0.92} \mathrm{Al}_{0.08}\right)_{12} \mathrm{O}_{22}$, generating two opposite directions of electric polarization. All these results demonstrate, on the microscopic level, that the electric field plays a role equivalent to that of the magnetic field in changing the clamped ferrimagnetic/ ferroelectric-domain configuration through the alignment of $\mathbf{P}$ and, consequently, $\mathbf{M}$, thus revealing the microscopic origin of the strong cross-coupling effect observed in this hexaferrite.

It is evident in Fig. 5b that the intensity of the NMR signal originating from the domains behaved quite differently from that of the signal originating from the domain walls. The NMR signal intensity always increased with increasing $E$, regardless of the poling direction. One possible explanation for this behaviour of the intensity originating from the domains is a change in the anisotropy field. The shrinkage of the coercive fields under the application of an $E$-bias in the $M-H$ curves presented in Fig. $2 \mathrm{~b}$ implies a small reduction of the magnetic anisotropy field. In such a case, the NMR signal is expected to be enhanced because magnetic anisotropy tends to fix the domain magnetisation along a certain direction, hindering magnetization rotation. Regardless of the mechanism, however, the change in the domain magnetization seems to be almost negligible compared with the gigantic converse $\mathrm{ME}$ coupling observed in $\mathrm{Ba}_{0.5} \mathrm{Sr}_{1.5} \mathrm{Zn}_{2}$ $\left(\mathrm{Fe}_{0.92} \mathrm{Al}_{0.08}\right)_{12} \mathrm{O}_{22}$ because the change is independent of the polarity of the applied electric field. Therefore, our NMR study demonstrates that the gigantic converse $\mathrm{ME}$ effect observed in $\mathrm{Ba}_{0.5} \mathrm{Sr}_{1.5} \mathrm{Zn}_{2}\left(\mathrm{Fe}_{0.92} \mathrm{Al}_{0.08}\right)_{12} \mathrm{O}_{22}$ was accompanied by domain-wall movement induced by the electric field, originating from the strong clamping of the ferroelectric and ferrimagnetic domains created in the transverse conical spin state. The results of the NMR measurements of the zero-field-cooled sample are also consistent with this clamping picture, as the NMR signal intensity in this case exhibited no change with $E$ within the experimental errors. It is because the longitudinal conical spin ordering (Fig. 1b), which forms after zero-field cooling, does not exhibit any ferroelectricity ${ }^{25}$.

Rapid switching speed of $\boldsymbol{M}$ induced by $\boldsymbol{E}$. The NMR results implying the movement of magnetic domain walls under an electric field seem to be unique to this insulating helimagnet. As in the case of domain-wall motion driven by spin-transfer torque $^{32}$, the domain-wall movement and the resultant changes in domain configuration under $E$ in this compound might also be sufficiently rapid for practical application. To test how rapidly the magnetic domains and/or domain walls respond to an electric field, we measured the resistance change of a giant magnetoresistance (GMR) sensor glued to the bottom surface of the crystal under a rapid triangular $E$ sweep, as depicted in Fig. 6a. We found that the GMR signals versus $E$ exhibited nearly the same shape as the $M-E$ loops presented in Fig. 3b, even when the $E$ sweep frequency was varied from 1 to $10 \mathrm{kHz}$ (Fig. 6b). 
When the sign of $E_{\mathrm{p}}$ was reversed, the GMR signal versus $E$ loops were modified accordingly, exhibiting behaviour very similar to that of the $M-E$ loops presented in Fig. 3b. When the GMR signal versus time is plotted alongside the triangular $E$ sweep applied on a time scale of $\sim 30 \mu \mathrm{s}(\sim 10 \mathrm{kHz})$, it is clear that the curve closely follows the shape of the rapid $E$ sweep (Fig. 6c). Although the speed of $E$ could not be further increased because of the limitations of the electronics, this result indicates that the switching time of $M$ induced by $E$ has an upper limit of $\sim 30 \mu$ s and may be much smaller than $30 \mu$ s. Our observation directly demonstrates that the speed of the domain-wall movement and the change in the domain configuration induced by $E$ may be sufficiently rapid to be useful in practical applications. The present results also suggest that the microscopic coupling between electric field and spin configuration may allow the more rapid, electrical control of $M$ in the frequency regime of giga or terahertz where the electromagnons of this helimagnet are located ${ }^{33}$

\section{Discussion}

It is worth discussing the major properties of $\mathrm{Ba}_{0.5} \mathrm{Sr}_{1.5} \mathrm{Zn}_{2}$ $\left(\mathrm{Fe}_{0.92} \mathrm{Al}_{0.08}\right)_{12} \mathrm{O}_{22}$ that create favourable conditions for such a large converse ME effect. One of the key characteristics is likely to be the soft ferrimagnetism with very weak in-plane anisotropy that exists in the transverse conical state of this hexaferrite (Supplementary Fig. 2). Unlike the conical spin state with an easy axis in $\mathrm{CoCr}_{2} \mathrm{O}_{4}$ (ref. 26), our $\mathrm{Ba}_{0.5} \mathrm{Sr}_{1.5} \mathrm{Zn}_{2}\left(\mathrm{Fe}_{0.92} \mathrm{Al}_{0.08}\right)_{12} \mathrm{O}_{22}$ has an easy $a b$ plane after the field-cooling process, and the sixfoldhexagonal anisotropic energy seems to be almost negligible in the easy plane. The smaller the magnetic anisotropy is, the smaller is the energy that is required for the application of $E$ to induce the simultaneous rotation of the $P$ and $M$ vectors inside the multiferroic domains or domain walls. Moreover, as implied in the ferroelectric phase diagram (Supplementary Fig. 1), the transverse conical state at zero magnetic field in this annealed $\mathrm{Ba}_{0.5} \mathrm{Sr}_{1.5} \mathrm{Zn}_{2}\left(\mathrm{Fe}_{0.92} \mathrm{Al}_{0.08}\right)_{12} \mathrm{O}_{22}$ seems to be stable up to $\sim 150 \mathrm{~K}$, below which temperature the converse $\mathrm{ME}$ effect becomes significant. Therefore, soft ferrimagnetism with minimal magnetic anisotropy and the related transverse conical spin state seems to be the most important factors leading to the observation of such large reversal and strong modulation of magnetization by an electric field. Furthermore, because other Y-type hexaferrites that exhibit a transverse conical state at higher temperatures exist, the mechanism and phenomena observed here are likely to be applicable even at room temperature ${ }^{23}$ and thus may pave the way towards eventually utilizing gigantic converse ME effects in real devices at room temperature.

\section{Methods}

Single-crystal growth and oxygen annealing. Y-type $\mathrm{Ba}_{0.5} \mathrm{Sr}_{1.5} \mathrm{Zn}_{2}\left(\mathrm{Fe}_{0.92} \mathrm{Al}_{0.08}\right)_{12}$ $\mathrm{O}_{22}$ single crystals were grown from a $\mathrm{Na}_{2} \mathrm{O}-\mathrm{Fe}_{2} \mathrm{O}_{3}$ flux in $\mathrm{air}^{34}$. The chemicals were mixed with a molar ratio of $\mathrm{BaCO}_{3}: \mathrm{SrCO}_{3}: \mathrm{ZnO}: \mathrm{Fe}_{2} \mathrm{O}_{3}: \mathrm{Al}_{2} \mathrm{O}_{3}: \mathrm{Na}_{2} \mathrm{O}=2.95$ : 16.74: 19.69: $49.32: 4.29: 7.01$ and melted at $1420^{\circ} \mathrm{C}$ in a Pt crucible, followed by several thermal cycles. The hexagonally-shaped single crystals of $\mathrm{Ba}_{0.5} \mathrm{Sr}_{1.5} \mathrm{Zn}_{2}\left(\mathrm{Fe}_{0.92} \mathrm{Al}_{0.08}\right)_{12} \mathrm{O}_{22}$ were all grown in the same batch. The $c$-axis parameters of the crystals were determined using X-ray diffraction. To increase the resistivity of the specimens, an optimized heat-treatment process was applied, in which the temperature was maintained at $900^{\circ} \mathrm{C}$ under flowing oxygen for 8 days, followed by cooling at a rate of $50^{\circ} \mathrm{Ch}^{-1}$ (ref. 35).

ME annealing procedures. The temperature and the magnetic field were controlled using a Physical Property Measurement System (PPMS; Quantum Design, USA). In all measurements, $H$ was applied along the [100] direction, and $E$ was applied along the [120] direction. For the electrical measurements, the samples were cut into a rectangular parallelepiped shape with the largest surface $\left(\sim 1 \mathrm{~mm}^{2}\right)$ normal to the [120] direction. Before the converse ME measurements, each specimen was subjected to an ME annealing procedure starting at $120 \mathrm{~K}$. For the ME annealing condition of $+H_{\mathrm{p}} \&+E_{\mathrm{p}}$, the sample was electrically poled at $E=220 \mathrm{kV} \mathrm{m}^{-1}$ upon changing the magnetic field from the paraelectric collinear state $\left(\mu_{0} H=2 \mathrm{~T}\right)$ to the ferrimagnetic state $\left(\mu_{0} H=1.2 \mathrm{~T}\right)$ at $120 \mathrm{~K}$. Then, with the same electric field and the magnetic field, the sample was initially cooled to the lowest investigated temperature $(15 \mathrm{~K})$. The $E$ was then turned off at $\mu_{0} H=1.2 \mathrm{~T}$, the electrodes were shorted, and the magnetic field was ramped down to zero prior to the converse ME experiments. For the converse ME experiments at higher temperatures, we sequentially increased the temperature after performing the lower-temperature experiments at zero magnetic field.

ME current measurements. The ME current was measured and integrated to determine the change in the electric polarization with the magnetic field. For the ME current measurement at $15 \mathrm{~K}$, a nearly identical ME annealing procedure was applied, except that the magnetic field was maintained at $1.2 \mathrm{~T}$ during the final stage. The ME current was then measured by sweeping $H$ either to the outside or to the inside of the ferroelectric region by passing through the zero-field region, that is, either down to $-2 \mathrm{~T}$ or up to $+2 \mathrm{~T}$ or first down to $-1.2 \mathrm{~T}$ and then up to $+2 \mathrm{~T}$. We also performed the equivalent converse ME and ME current experiments under the $\mathrm{ME}$ annealing condition of $+H_{\mathrm{p}},-E_{\mathrm{p}}$ in a similar manner.

Magnetization under an electric field. Magnetization curves were measured using a vibrating sample magnetometer (VSM) in a PPMS (Quantum Design). The sample was cooled from room temperature to $15 \mathrm{~K}$ at $\mu_{0} H=2 \mathrm{~T}$ for the magnetization measurements after field cooling. For the magnetization measurements under an electric-field bias, we cooled the sample after applying the ME annealing procedure starting from $120 \mathrm{~K}$ as described above, and the target electric field was applied at $15 \mathrm{~K}$ and $1.2 \mathrm{~T}$ before ramping down the magnetic field. For the low- $H$ measurements $\left(\left|\mu_{0} H\right| \leq 2 \mathrm{mT}\right)$ in particular, we carefully calibrated the $H$ of the superconducting magnet in the PPMS using a standard paramagnetic Pd sample (Quantum Design Application Note 1070-207). For the magnetization measurements under $E$, we modified the conventional VSM sample holder to permit the application of high $E$.

P-E loop measurements. The $P-E$ loop of the sample was measured using the Positive-Up-Negative-Down (PUND) method in our homemade setup to extract only the hysteretic parts of the change in the electric polarization ${ }^{36}$. Triangular electric pulses with a duration of $0.5 \mathrm{~ms}$ were applied, using a constant peak amplitude for each pulse.

Ferroelectric phase diagram of the annealed crystal. For Y-type multiferroic hexaferrites, oxygen annealing can slightly change the magnetic and ferroelectric phase boundaries ${ }^{35}$. Therefore, we determined the ferroelectric phase diagram of the studied crystals, in which the magnetization behaviour versus temperature indicated long-range antiferromagnetic ordering at the Néel temperature $T_{\mathrm{N}} \sim 260 \mathrm{~K}$. It is known that the ferroelectric phase diagram can be determined by measuring the dielectric anomaly (peak) as a function of the magnetic field under isothermal conditions ${ }^{16}$. By mapping out the peak field positions in the dielectric constant versus the magnetic field at selected temperatures, we plotted the ferroelectric phase diagram of the annealed crystals, as presented in Supplementary Fig. 1. From this study, we found that the dielectric-constant peaks not only originate from switching at the paraelectric-to-ferroelectric phase boundary, but also appear at zero magnetic field upon the occurrence of field-induced ferroelectric-domain switching. In the transverse conical state that formed at zero $H$-bias after ME annealing, we observed a characteristic peak at zero magnetic field attributable to ferroelectric-domain switching up to $\sim 150 \mathrm{~K}$, in addition to the high-field peaks located above $1 \mathrm{~T}$ corresponding to the crossing of the paraelectric phase boundary.

At $\sim 150 \mathrm{~K}$, the low-field peak slightly increased in magnetic field towards the $10-20 \mathrm{mT}$ scale, and then, at higher temperatures, the peak structure broadened, indicating that the transverse conical state was only stable below $150 \mathrm{~K}$ at zero field From a neutron-diffraction study ${ }^{25}$, we found that above $150 \mathrm{~K}$, the alternating longitudinal conical state is stable up to around $\sim 230 \mathrm{~K}$ at zero magnetic field. Between $T_{\mathrm{N}}$ and $230 \mathrm{~K}$, there seems to be a mixed phase that is a combination of a modified helix and alternating longitudinal conical states. Therefore, above $\sim 150 \mathrm{~K}$, there is no ferroelectric phase at zero magnetic field, and only a fieldinduced crossover from a paraelectric phase (longitudinal conical or modified helix phase) to a ferroelectric (transverse conical) phase seems to be gradually appearing.

Magnetic anisotropy in $\mathrm{Ba}_{\mathbf{0 . 5}} \mathrm{Sr}_{\mathbf{1 . 5}} \mathbf{Z n}_{\mathbf{2}}\left(\mathrm{Fe}_{\mathbf{0 . 9 2}} \mathrm{Al}_{\mathbf{0 . 0 8}}\right)_{\mathbf{1 2}} \mathbf{O}_{\mathbf{2 2}}$. The transverse conical spin configuration in the $\mathrm{Ba}_{0.5} \mathrm{Sr}_{1.5} \mathrm{Zn}_{2}\left(\mathrm{Fe}_{0.92} \mathrm{Al}_{0.08}\right)_{12} \mathrm{O}_{22}$ crystal has magnetic anisotropy of the easy-plane type in which magnetic anisotropy is very small. This feature can be demonstrated by handling the cone axis with a small rotating magnetic field of several tens of $\mathrm{mT}^{17}$. We demonstrated the angular dependence of $P$ under rotating $H$ of $20 \mathrm{mT}, 0.2 \mathrm{~T}$ and $1 \mathrm{~T}$ applied in the $a b$ plane, as illustrated in Supplementary Fig. 2. All $P$-values followed nearly sinusoidal curves, strongly suggesting that the transverse cone axis follows the direction of $H$, while the spin helicity remains intact. The highly tunable $P$ under the rotating $H$ as small as $20 \mathrm{mT}$ highlights the small magnetic anisotropy in the $a b$ plane. 
NMR measurements. The ${ }^{57} \mathrm{Fe}$ NMR spectra were obtained using our homemade spectroscopy setup and a standard spin-echo $(\pi / 2-\pi)$ sequence. For $H$ and $E$ dependences of $I_{\max }$, the frequency of $H_{1}$ was $69 \mathrm{MHz}$, which was the central frequency of the main peak ${ }^{37}$. The ME annealing procedure for $+H_{\mathrm{p}} \&+E_{\mathrm{p}}$ was done as follows: the sample was cooled to $130 \mathrm{~K}$ in the absence of both $H$ and $E$. At $130 \mathrm{~K}$, the sample was electrically poled at $E=250 \mathrm{kV} \mathrm{m}^{-1}$ while changing $\mu_{0} H$ from $0 \mathrm{~T}$ to $0.4 \mathrm{~T}$. Then, the sample was cooled to $7 \mathrm{~K}$ while both $E$ and $H$ turned on

GMR sensor measurements. The GMR chip sensor (NVE Sensors, AA004-02) was calibrated at $15 \mathrm{~K}$ before we applied the electric field to the sample. To ensure operation in the linear region of the GMR sensor, a bias $H$ of $2 \mathrm{mT}$ was applied. Meanwhile, a constant excitation voltage of $0.4 \mathrm{~V}$ was applied to the GMR sensor during the measurements. The configuration of the sample was chosen such that the change in the direction of $M$ was parallel to the $H$-sensitive direction of the GMR sensor.

\section{References}

1. Spaldin, N. A. \& Fiebig, M. The renaissance of magnetoelectric multiferroics. Science 309, 391-392 (2005).

2. Cheong, S.-W. \& Mostovoy, M. Multiferroics: a magnetic twist for ferroelectricity. Nat. Mater. 6, 13-20 (2007).

3. Ramesh, R. \& Spaldin, N. A. Multiferroics: progress and prospects in thin films. Nat. Mater. 6, 21-29 (2007).

4. Eerenstein, W., Mathur, N. D. \& Scott, J. F. Multiferroic and magnetoelectric materials. Nature 442, 759-765 (2006).

5. Folen, V. J., Rado, G. T. \& Stalder, E. W. Anisotropy of the magnetoelectric effect in $\mathrm{Cr}_{2} \mathrm{O}_{3}$. Phys. Rev. Lett. 6, 607-608 (1961).

6. Ascher, E., Riedel, H., Schmid, H. \& Stössel, H. Some properties of ferromagnetoelectric nickel-iodine boracite, $\mathrm{Ni}_{3} \mathrm{~B}_{7} \mathrm{O}_{13}$ I. J. Appl. Phys. 37, 1404 (1966).

7. Brown, Jr W. F., Hornreich, R. M. \& Shtrikman, S. Upper bound on the magnetoelectric susceptibility. Phys. Rev. 168, 574-577 (1968).

8. Tokunaga, Y. et al. Composite domain walls in a multiferroic perovskite ferrite. Nat. Mater. 8, 558-562 (2009).

9. Choi, Y. J., Zhang, C. L., Lee, N. \& Cheong, S.-W. Cross-control of magnetization and polarization by electric and magnetic fields with competing multiferroic and weak-ferromagnetic phases. Phys. Rev. Lett. 105, 097201 (2010).

10. Chun, S. H. et al. Electric field control of nonvolatile four-state magnetization at room temperature. Phys. Rev. Lett. 108, 177201 (2012).

11. Heron, J. T. et al. Electric-field-induced magnetization reversal in a ferromagnet-multiferroic heterostructure. Phys. Rev. Lett. 107, 217202 (2011).

12. Tokunaga, Y., Taguchi, Y., Arima, T. H. \& Tokura, Y. Electric-field-induced generation and reversal of ferromagnetic moment in ferrites. Nat. Phys. 8, 838-844 (2012).

13. Iyama, A. \& Kimura, T. Magnetoelectric hysteresis loops in $\mathrm{Cr}_{2} \mathrm{O}_{3}$ at room temperature. Phys. Rev. B 87, 180408(R) (2013).

14. Pullar, R. C. Hexagonal ferrites: A review of the synthesis, properties and applications of hexaferrite ceramics. Prog. Mater. Sci. 57, 1191-1334 (2012).

15. Kimura, T. Magnetoelectric hexaferrites. Annu. Rev. Condens. Matter Phys. 3, 93-110 (2012)

16. Kimura, T., Lawes, G. \& Ramirez, A. P. Electric polarization rotation in a hexaferrite with long-wavelength magnetic structures. Phys. Rev. Lett. 94, 137201 (2005)

17. Ishiwata, S. et al. Low-magnetic-field control of electric polarization vector in a helimagnet. Science 319, 1643-1646 (2008).

18. Chun, S. H. et al. Realization of giant magnetoelectricity in helimagnets. Phys. Rev. Lett. 104, 037204 (2010).

19. Tokunaga, Y. et al. Multiferroic M-type hexaferrites with a room temperature conical state and magnetically controllable spin helicity. Phys. Rev. Lett. 105, 257201 (2010)

20. Kitagawa, Y. et al. Low-field magnetoelectric effect at room temperature. Nat. Mater. 9, 797-802 (2010)

21. Okumura, K. et al. Magnetism and magnetoelectricity of a U-type hexaferrite $\mathrm{Sr}_{4} \mathrm{Co}_{2} \mathrm{Fe}_{36} \mathrm{O}_{60}$. Appl. Phys. Lett. 98, 212504 (2011).
22. Song, Y. Q. et al. Spin reorientation transition and near room-temperature multiferroic properties in a W-type hexaferrite $\mathrm{SrZn}_{1.15} \mathrm{Co}_{0.85} \mathrm{Fe}_{16} \mathrm{O}_{27}$. J. Appl. Phys. 115, 093905 (2014).

23. Hirose, S., Haruki, K., Ando, A. \& Kimura, T. Mutual control of magnetization and electrical polarization by electric and magnetic fields at room temperature in Y-type $\mathrm{BaSrCo}_{2-\mathrm{x}} \mathrm{Zn}_{\mathrm{x}} \mathrm{Fe}_{11} \mathrm{AlO}_{22}$ ceramics. Appl. Phys. Lett. 104, 022907 (2014).

24. Smit, J. \& Wijn, H. P. J. Ferrites (Philips Technical Library, 1959).

25. Lee, H. B. et al. Field-induced incommensurate-to-commensurate phase transition in the magnetoelectric hexaferrite $\mathrm{Ba}_{0.5} \mathrm{Sr}_{1.5} \mathrm{Zn}_{2}\left(\mathrm{Fe}_{1-\mathrm{x}} \mathrm{Al}_{\mathrm{x}}\right)_{12} \mathrm{O}_{22}$ Phys. Rev. B 83, 144425 (2011).

26. Yamasaki, Y. et al. Magnetic reversal of the ferroelectric polarization in a multiferroic spinel oxide. Phys. Rev. Lett. 96, 207204 (2006).

27. Katsura, H., Nagaosa, N. \& Balatsky, A. V. Spin current and magnetoelectric effect in noncollinear magnets. Phys. Rev. Lett. 95, 057205 (2005).

28. Mostovoy, M. Ferroelectricity in spiral magnets. Phys. Rev. Lett. 96, 067601 (2006).

29. Turov, E. A. \& Petrov, M. P. Nuclear Magnetic Resonance in Ferro- and Antiferromagnets (Wiley, 1972).

30. Riedi, P. C. Magnetic studies with zero field NMR. Hyp. Int. 49, 335-356 (1989).

31. Obol, M. \& Vittoria, C. Microwave permeability of Y-type hexaferrites in zero field. J. Appl. Phys. 94, 4013-4017 (2003).

32. Parkin, S. S. P., Hayashi, M. \& Thomas, L. Magnetic domain-wall racetrack memory. Science 320, 190-194 (2008).

33. Kida, N. et al. Electric-dipole-active magnetic resonance in the conical-spin magnet $\mathrm{Ba}_{2} \mathrm{Mg}_{2} \mathrm{Fe}_{12} \mathrm{O}_{22}$. Phys. Rev. B 80, 220406(R) (2009).

34. Momozawa, N., Mita, M. \& Takei, H. Single crystal growth of $\left(\mathrm{Ba}_{1-} \mathrm{Sr}_{\mathrm{x}}\right)_{2} \mathrm{Zn}_{2} \mathrm{Fe}_{12} \mathrm{O}_{22}$ from high temperature solution. J. Cryst. Growth 83, 403-409 (1987)

35. Chai, Y. S. et al. Low-magnetic-field control of dielectric constant at room temperature realized in $\mathrm{Ba}_{0.5} \mathrm{Sr}_{1.5} \mathrm{Zn}_{2} \mathrm{Fe}_{12} \mathrm{O}_{22}$. New J. Phys. 11, 073030 (2009).

36. Chai, Y. S. et al. Intrinsic ferroelectric polarization of orthorhombic manganites with E-type spin order. Phys. Rev. B 85, 184406 (2012).

37. Kwon, S. et al. ${ }^{57} \mathrm{Fe}$ NMR study of the magnetoelectric hexaferrite $\mathrm{Ba}_{0.5} \mathrm{Sr}_{1.5} \mathrm{Zn}_{2} \mathrm{Fe}_{12} \mathrm{O}_{22}$ and $\mathrm{Ba}_{0.5} \mathrm{Sr}_{1.5} \mathrm{Zn}_{2}\left(\mathrm{Fe}_{0.92} \mathrm{Al}_{0.08}\right)_{12} \mathrm{O}_{22}$. Phys. Rev. B 88, 064404 (2013).

\section{Acknowledgements}

The work at Seoul National University was supported by the Creative Research Initiative (2010-0018300). The work at KAIST was supported by the National Research Foundation (Grant Nos NRF-2009-0078342 and 2012R1A2A2A01003598).

\section{Author contributions}

Y.S.C., S.H.C. and K.H.K. designed and initiated this study. S.H.C. grew the single crystals, and Y.S.C. and S.H.C. conducted the sample preparation for each experiment. I.K., S.H.C. and Y.S.C. constructed the setup for VSM measurements under an electric field. Y.S.C. performed the VSM measurements under an electric field. Y.S.C. and B.-G.J. performed the GMR sensor measurements. Y.S.C. and S.H.C. performed the conventional VSM measurements. S.K. and S.L. performed the NMR measurements and discussed the data with Y.S.C., S.H.C. and K.H.K.; Y.S.C., S.K., S.H.C., K.H.K. and S.L. wrote the paper.

\section{Additional information}

Supplementary Information accompanies this paper at http://www.nature.com/ naturecommunications

Competing financial interests: The authors declare no competing financial interest.

Reprints and permission information is available online at http://npg.nature.com/ reprintsandpermissions/

How to cite this article: Chai, Y. S. et al. Electrical control of large magnetization reversal in a helimagnet. Nat. Commun. 5:4208 doi: 10.1038/ncomms5208 (2014). 